\title{
A generalized scheme based on shifted Jacobi polynomials for numerical simulation of coupled systems of multi-term fractional-order partial differential equations
}

\author{
Kamal Shah, Hammad Khalil and Rahmat Ali Khan
}

\begin{abstract}
Due to the increasing application of fractional calculus in engineering and biomedical processes, we analyze a new method for the numerical simulation of a large class of coupled systems of fractional-order partial differential equations. In this paper, we study shifted Jacobi polynomials in the case of two variables and develop some new operational matrices of fractional-order integrations as well as fractional-order differentiations. By the use of these operational matrices, we present a new and easy method for solving a generalized class of coupled systems of fractionalorder partial differential equations subject to some initial conditions. We convert the system under consideration to a system of easily solvable algebraic equation without discretizing the system, and obtain a highly accurate solution. Also, the proposed method is compared with some other well-known differential transform methods. The proposed method is computer oriented. We use MatLab to perform the necessary calculation. The next two parts will appear soon.
\end{abstract}

\section{Introduction}

It is well known that coupled systems of partial differential equations (PDEs) are widely used in engineering and biomechanics problems. Some applications of coupled systems of PDEs arise in biomechanics when modeling the electrical activity in the heart (see, for example, $[30,53,54,59])$. They also occur when modeling some chemical and material engineering problems, such as a system containing a continuous stirred tank reactor (CSTR) and a plug flow reactor $(\mathrm{PFR})$ in series $[\mathbf{1}, \mathbf{3 5}]$. Various applications are found in solid mechanics; for example, the dynamics of multi-deformable bodies coupled by standard light fractional-order discrete continuum layers is described by coupled partial fractional-order differential equations $[10,20,40,47]$. Coupled systems of PDEs also appear in the modeling of some important electromagnetic and gravitational problems (see, for example, $[\mathbf{2 9}, \mathbf{5 6}]$ ).

Very recently, fractional calculus and fractional differential equations have gained the attention of scientists and many interesting applications in several different disciplines $[15,17,38,46]$ have been investigated. Several authors have found that many engineering and physical processes can be modeled and explained well by systems of fractional-order differential equations, compared with systems of conventional differential equations, and also that fractional-order differentials and integrals provide more a accurate and real insight into systems under consideration (see, for example, $[\mathbf{1 3}, \mathbf{1 4}, \mathbf{1 6}-\mathbf{2 0}, \mathbf{3 1}, \mathbf{3 3}, \mathbf{3 4}, \mathbf{4 8}, \mathbf{5 5}]$ and the references therein). All of these engineering problems are also mathematical problems and are described by PDEs with integer or fractional-order derivative terms which can be discretized into a problem of solving a system of ordinary differential, integro-differential or fractionalorder partial differential equations.

The analytical results based on the existence and uniqueness of solutions to some fractional differential equations have been investigated by many authors (see, for example, $[\mathbf{2 5}, \mathbf{4 5}$, 50-52] and the references therein). Bearing in mind the increasing application of fractional-

Received 11 May 2015; revised 21 October 2016.

2010 Mathematics Subject Classification 35R11 (primary), 65M06 (secondary). 
order differential equations and PDEs, and due to the computational complexities of fractional calculus and the non-availability of their explicit analytic solutions, the need to exploit various efficient and reliable numerical schemes is a problem of fundamental interest.

In the literature, a number of numerical methods have been proposed for obtaining approximate solutions to fractional-order differential equations such as eigenvector expansion, the Adomian decomposition method (ADM), the fractional differential transform method (FDTM) $[\mathbf{2}, \mathbf{8}]$ and the generalized block-pulse operational matrix method [32], to name but a few. In $[\mathbf{5}, \mathbf{6}]$, some linear and nonlinear fractional-order PDEs have been solved by using the homotopy analysis method (HAM). Very recently, in [7], the authors constructed two high-order algorithms to handle multi-term time fractional diffusion-wave equations. Also, some interesting numerical schemes based on operational matrices of fractional-order integration with Haar wavelets, Legendre wavelets, sine-cosine and Chebyshev wavelets for solutions to fractional-order differential equations have been developed in $[\mathbf{2 8}, \mathbf{3 9}, \mathbf{4 2 - 4 4}, \mathbf{5 7}]$. In continuation, a new numerical scheme, based on the Haar wavelet, involving an operational matrix of integration is developed in [4] for solutions of fractional-order multi-point boundary value problems. Some new results related to the Jacobi polynomials and operational matrices have been recently discovered (see [22-24]). Some operational matrices of arbitrary-order derivatives and integrals and their applications are also constructed by using B-spline functions, fractional Jacobi functions and the Taylor series method (for details, see $[\mathbf{9}, \mathbf{2 1}, \mathbf{2 7}]$ ).

All the operational matrix methods are used to solve fractional-order differential equations and PDEs. We attempt to generalize the operational matrix technique to solve coupled systems of fractional-order PDEs. In this paper, we use Jacobi polynomials in two variables and develop new operational matrices of fractional-order differentiations and integrations to solve a more generalized class of coupled systems of fractional-order PDEs of the form

$$
\begin{aligned}
& \frac{\partial^{\sigma} U(x, y)}{\partial x^{\sigma}}=\sum_{i=0}^{n} a_{i} \frac{\partial^{i} U(x, y)}{\partial y^{i}}+\sum_{i=0}^{n} b_{i} \frac{\partial^{i} V(x, y)}{\partial x^{i}}+\sum_{i=0}^{n} c_{i} \frac{\partial^{i} V(x, y)}{\partial y^{i}}+F_{1}(x, y), \\
& \frac{\partial^{\sigma} V(x, y)}{\partial x^{\sigma}}=\sum_{i=0}^{n} d_{i} \frac{\partial^{i} V(x, y)}{\partial y^{i}}+\sum_{i=0}^{n} e_{i} \frac{\partial^{i} U(x, y)}{\partial x^{i}}+\sum_{i=0}^{n} f_{i} \frac{\partial^{i} U(x, y)}{\partial y^{i}}+F_{2}(x, y),
\end{aligned}
$$

subject to the initial conditions

$$
U^{(i)}(0, y)=H_{i}(y), \quad V^{(i)}(0, y)=G_{i}(y), \quad i=0,1, \ldots, n,
$$

where $n-1 \leqslant \sigma<n, a_{i}, b_{i}, c_{i}, d_{i}, e_{i}, f_{i}$ are all real constants, $U=U(x, y)$ and $V=V(x, y)$ are the unknown solution of the system to be determined and $U(x, y), V(x, y), F_{1}(x, y)$ and $F_{2}(x, y)$ $\in C^{n}([0, \eta] \times[0, \eta])$.

The method reduces the system of fractional-order differential equations to a coupled system of algebraic equations. Generally, large systems of algebraic equations may lead to greater computational complexity and large storage requirements. However, our technique is simple and reduces the computational complexity of the resulting algebraic system. It is worth mentioning here that the proposed method is computer oriented, and it is designed in such way that it can be easily simulated with any computational software.

The rest of article is organized as follows. We begin by introducing some necessary definitions and mathematical preliminaries from fractional calculus and Jacobi polynomials. In Section 3, operational matrices of fractional-order derivatives and fractional-order integrals are developed. Section 4 is devoted to the application of operational matrices of fractional derivatives and fractional integrals to solve a coupled system of fractional-order PDEs. In Section 5, the proposed method is applied to several examples and the results are discussed and also compared with some other methods. The last section is devoted to a conclusion and a brief overview of future work. 


\section{Preliminaries}

For convenience, this section summarizes some concepts, definitions and basic results from fractional calculus, which are useful for further development in this paper.

Definition 2.1. $[\mathbf{2 6}, \mathbf{4 1}]$ Given an interval $[a, b] \subset \mathbb{R}$, the Riemann-Liouville fractionalorder integral of order $\alpha \in \mathbb{R}_{+}$of a function $\phi \in\left(L^{1}[a, b], \mathbb{R}\right)$ is defined by

$$
\mathcal{I}_{a+}^{\alpha} \phi(t)=\frac{1}{\Gamma(\alpha)} \int_{a}^{t}(t-s)^{\alpha-1} \phi(s) d s,
$$

provided that the integral on right-hand side exists.

REMARK 2.2. It is to be noted that, throughout this paper, we will use the Caputo fractionalorder derivative.

Definition 2.3. For a given function $\phi(x) \in C^{n}[a, b]$, the Caputo fractional-order derivative of order $\alpha$ is defined as

$$
D^{\alpha} \phi(x)=\frac{1}{\Gamma(n-\alpha)} \int_{a}^{x} \frac{\phi^{(n)}(t)}{(x-t)^{\alpha+1-n}} d t, \quad n-1 \leqslant \alpha<n, n \in N,
$$

provided that the right-hand side is pointwise defined on $(a, \infty)$, where $n=[\alpha]+1$.

Hence, it follows that

$$
D^{\alpha} x^{k}=\frac{\Gamma(1+k)}{\Gamma(1+k-\alpha)} x^{k-\alpha}, \quad I^{\alpha} x^{k}=\frac{\Gamma(1+k)}{\Gamma(1+k+\alpha)} x^{k+\alpha} \quad \text { and } \quad D^{\alpha} C=0, \text { for a constant } C \text {. }
$$

\subsection{The shifted Jacobi polynomials}

The well-known two parametric Jacobi polynomials defined on $[0, \eta]$, with parameter $\alpha, \beta$ are given by the relation

$$
P_{\eta, i}^{(\alpha, \beta)}(x)=\sum_{k=0}^{i} \frac{(-1)^{i-k} \Gamma(i+\beta+1) \Gamma(i+k+\alpha+\beta+1)}{\Gamma(k+\beta+1) \Gamma(i+\alpha+\beta+1) \Gamma(i-k+1) \Gamma(k+1) \eta^{k}} x^{k}, \quad i=0,1,2 \ldots
$$

These polynomials are orthogonal and the orthogonality relation is

$$
\int_{0}^{\eta} P_{\eta, i}^{(\alpha, \beta)}(x) P_{\eta, j}^{(\alpha, \beta)}(x) W_{\eta}^{(\alpha, \beta)}(x) d x=R_{\eta, j}^{(\alpha, \beta)} \delta_{i, j},
$$

where

$$
W_{\eta}^{(\alpha, \beta)}(x)=(\eta-x)^{\alpha} x^{\beta}
$$

is the weight function. Also,

$$
R_{\eta, j}^{(\alpha, \beta)} \delta_{i, j}=\frac{\eta^{\alpha+\beta+1} \Gamma(j+\alpha+1) \Gamma(j+\beta+1)}{(2 j+\alpha+\beta+1) \Gamma(j+1) \Gamma(j+\alpha+\beta+1)} .
$$

This implies that any function $v(x)$ that is square integrable in $[0, \eta]$ can be approximated by shifted Jacobi polynomials, that is,

$$
v(x) \approx \sum_{a=0}^{m} C_{a} P_{\eta, j}^{(\alpha, \beta)}(x),
$$


and as $m \rightarrow \infty$, the approximation becomes equal to the exact function. By using (5), (6) and (7), we can easily calculate the coefficient $C_{a}$. We can also write (8) in vector form as

$$
v(x) \approx H_{M}^{T} \hat{\Psi}_{M}(x),
$$

where $M=m+1, H_{M}$ is the coefficient vector and $\hat{\Psi}_{M}(x)$ is an $M$ term vector function. We extend the notion to two-dimensional space and define two-dimensional Jacobi polynomials of order $M$ on the region $[0, \eta] \times[0, \eta]$ as a product function of two Jacobi polynomials

$$
P_{\eta, n}^{(\alpha, \beta)}(x, y)=\left(P_{\eta, i}^{(\alpha, \beta)}(x)\right)\left(P_{\eta, j}^{(\alpha, \beta)}(y)\right), \quad n=M i+j+1, i=0,1,2, \ldots, m, j=0,1,2, \ldots, m .
$$

The orthogonality condition of $P_{\eta, n}^{(\alpha, \beta)}(x, y)$ is found to be

$$
\begin{aligned}
& \int_{0}^{\eta} \int_{0}^{\eta}\left(P_{\eta, a}^{(\alpha, \beta)}(x)\right)\left(P_{\eta, b}^{(\alpha, \beta)}(y)\right)\left(P_{\eta, c}^{(\alpha, \beta)}(x)\right)\left(P_{\eta, d}^{(\alpha, \beta)}(x)\right) W_{\eta}^{(\alpha, \beta)}(x) W_{\eta}^{(\alpha, \beta)}(y) d x d y \\
& \quad=R_{\eta, c}^{(\alpha, \beta)} \delta_{a, c} R_{\eta, d}^{(\alpha, \beta)} \delta_{b, d} .
\end{aligned}
$$

Any function that is square integrable in $[0, \eta] \times[0, \eta]$ can be approximated by $M$ terms of the Jacobi polynomials $P_{\eta, n}^{(\alpha, \beta)}(x, y)$, that is,

$$
f(x, y) \approx \sum_{a=0}^{m} \sum_{b=0}^{m} C_{a b}\left(P_{\eta, a}^{(\alpha, \beta)}(x)\right)\left(P_{\eta, b}^{(\alpha, \beta)}(y)\right),
$$

where $C_{a b}$ can be obtained by the relation

$$
C_{a b}=\frac{1}{R_{\eta, a}^{(\alpha, \beta)} R_{\eta, b}^{(\alpha, \beta)}} \int_{0}^{\eta} \int_{0}^{\eta} f(x, y)\left(P_{\eta, a}^{(\alpha, \beta)}(x)\right)\left(P_{\eta, b}^{(\alpha, \beta)}(y)\right) W_{\eta}^{(\alpha, \beta)}(x, y) d x d y .
$$

The weight function is defined as

$$
W_{\eta}^{(\alpha, \beta)}(x, y)=W_{\eta}^{(\alpha, \beta)}(x) W_{\eta}^{(\alpha, \beta)}(y) .
$$

For simplicity, we use the notation $C_{n}=C_{a b}$, where $n=M a+b+1$, and rewrite (12) as

$$
f(x, y) \approx \sum_{n=1}^{M^{2}} C_{n} P_{\eta, n}^{(\alpha, \beta)}(x, y)=K_{M^{2}}^{T} \Psi_{M^{2}}(x, y)
$$

in vector notation, where $K_{M^{2}}$ is an $M^{2} \times 1$ coefficient column vector and $\Psi_{M^{2}}(x, y)$ is an $M^{2} \times 1$ column vector of functions defined by

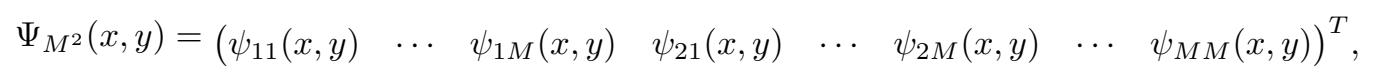

where $\psi_{i+1, j+1}(x, y)=\left(P_{\eta, i}^{(\alpha, \beta)}(x)\right)\left(P_{\eta, j}^{(\alpha, \beta)}(y)\right), i, j=0,1,2, \ldots, m$.

\subsection{Error analysis}

In this section, we provide an analytic expression for the error of approximation of a sufficiently smooth function $g(x, y)$ on $\Delta=[0, \eta] \times[0, \eta]$. For this purpose, let $\prod_{M, M}(x, y)$ be the space of Jacobi polynomials. We assume that $g_{(M, M)}(x, y)$ is the best approximation in $\prod_{(M, M)}(x, y)$. For this purpose, consider a polynomial $\hat{P}_{(M, M)}(x, y)$ that is any polynomial of degree $\leqslant M$ in variables $x$ and $y$, respectively. Then, from the definition of best approximation,

$$
\left\|g(x, y)-g_{(M, M)}(x, y)\right\|_{2} \leqslant\left\|g(x, y)-P_{(M, M)}(x, y)\right\|_{2} .
$$


The inequality in (16) is also satisfied if $P_{(M, M)}(x, y)$ is interpolating the polynomial at point $\left(x_{i}, y_{j}\right)$ and then, by a similar argument as in [12], the error of the approximation is given by

$$
\left\|g(x, y)-P_{(M, M)}(x, y)\right\|_{2} \leqslant\left(C_{1}+C_{2}+C_{3} \frac{1}{M^{M+1}}\right) \frac{1}{M^{M+1}}
$$

where

$$
\begin{aligned}
C_{1} & =\frac{1}{4} \max _{(x, y) \in[0,1] \times[0,1]}\left|\frac{\partial^{M+1}}{\partial x^{M+1}} g(x, y)\right|, \quad C_{2}=\frac{1}{4} \max _{(x, y) \in[0,1] \times[0,1]}\left|\frac{\partial^{M+1}}{\partial y^{M+1}} g(x, y)\right| \\
C_{3} & =\frac{1}{16} \max _{(x, y) \in[0,1] \times[0,1]}\left|\frac{\partial^{2 M+2}}{\partial x^{M+1} \partial y^{M+1}} g(x, y)\right| .
\end{aligned}
$$

We refer the reader to [37] for the proof of the above result.

\section{Operational matrices of integrations and differentiations}

Spectral methods are very strong tools for solving many kinds of differential equations arising in various fields of science and engineering. The aforementioned methods are based on various kinds of operational matrices. The construction and application of the operational matrices has recently become a fast-growing topic for research. In the case of a single variable, the operational matrices of fractional-order integration and differentiation are available in $[42,49]$. Similarly, operational matrices for two-dimensional orthogonal polynomials can be found in $[\mathbf{2 2 - 2 4}]$. We generalize the notion to the case of two variables and develop operational matrices of fractional-order integrations and differentiations. The construction of these operational matrices are analogous. In order to make this article a self-contained material, we present the proof and construction of operational matrices for two-dimensional Jacobi polynomials.

Lemma 3.1. Let $\Psi_{M^{2}}(x, y)$ be as defined in (15). Then the integration of order $v$ of $\Psi_{M^{2}}(x, y)$ with respect to $x$ is given by

$$
I_{x}^{v}\left(\Psi_{M^{2}}(x, y)\right) \simeq P_{M^{2} \times M^{2}}^{v, x} \Psi_{M^{2}}(x, y),
$$

where $P_{M^{2} \times M^{2}}^{v, x}$ is the operational matrix of integration of order $v$ and is defined as

$$
P_{M^{2} \times M^{2}}^{v, x}=\left(\begin{array}{cccccc}
\Omega_{1,1, k} & \Omega_{1,2, k} & \cdots & \Omega_{1, r, k} & \cdots & \Omega_{1, M^{2}, k} \\
\Omega_{2,1, k} & \Omega_{2,2, k} & \cdots & \Omega_{2, r, k} & \cdots & \Omega_{2, M^{2}, k} \\
\vdots & \vdots & \vdots & \vdots & \vdots & \vdots \\
\Omega_{q, 1, k} & \Omega_{q, 2, k} & \cdots & \Omega_{q, r, k} & \cdots & \Omega_{q, M^{2}, k} \\
\vdots & \vdots & \vdots & \vdots & \vdots & \vdots \\
\Omega_{M^{2}, 1, k} & \Omega_{M^{2}, 2, k} & \cdots & \Omega_{M^{2}, r, k} & \cdots & \Omega_{M^{2}, M^{2}, k}
\end{array}\right),
$$

and $r=M i+j+1, q=M a+b+1, \Omega_{q, r, k}=\Theta_{i, j, a, b, k}$ for $i, j, a, b=0,1,2, \ldots, m$,

$$
\Theta_{i, j, a, b, k}=\sum_{k=0}^{a} \Lambda_{a, k, v} S_{i, j, b},
$$




$$
\begin{aligned}
S_{i j b}= & \delta_{j, b} \sum_{l=0}^{i} \\
& \times \frac{(-1)^{i-l}(2 i+\alpha+\beta+1) \Gamma(i+1) \Gamma(i+l+\alpha+\beta+1) \Gamma(k+v+l+\beta+1) \Gamma(\alpha+1) \eta^{v}}{\Gamma(i+\alpha+1) \Gamma(l+\beta+1) \Gamma(i-l+1) \Gamma(l+1) \Gamma(k+v+l+\beta+\alpha+2)} .
\end{aligned}
$$

Also,

$$
\Lambda_{a, k, v}=\frac{(-1)^{a-k} \Gamma(a+\beta+1) \Gamma(a+k+\alpha+\beta+1) \Gamma(1+k)}{\Gamma(k+\beta+1) \Gamma(a+\alpha+\beta+1) \Gamma(a-k+1) \Gamma(k+1) \Gamma(1+k+v) \eta^{k}} .
$$

Proof. In order to prove the result, take $P_{\eta, n}^{(\alpha, \beta)}(x, y)$ as defined by (10). Then the fractional integral of order $v$ of $P_{\eta, n}^{(\alpha, \beta)}(x, y)$ with respect to $x$ is given by

$$
\begin{aligned}
I_{x}^{v} P_{\eta, n}^{(\alpha, \beta)}(x, y) & =I_{x}^{v} P_{\eta, a}^{(\alpha, \beta)}(x) P_{\eta, b}^{(\alpha, \beta)}(y) \\
& =\sum_{k=0}^{a} \frac{(-1)^{a-k} \Gamma(a+\beta+1) \Gamma(a+k+\alpha+\beta+1)}{\Gamma(k+\beta+1) \Gamma(a+\alpha+\beta+1) \Gamma(a-k+1) \Gamma(k+1) \eta^{k}} I_{x}^{v} x^{k} P_{\eta, a}^{(\alpha, \beta)}(y),
\end{aligned}
$$

which, in view of the definition of fractional integrals, takes the form

$$
\begin{aligned}
& I_{x}^{v} P_{\eta, a}^{(\alpha, \beta)}(x) P_{\eta, b}^{(\alpha, \beta)}(y) \\
& \quad=\sum_{k=0}^{a} \frac{(-1)^{a-k} \Gamma(a+\beta+1) \Gamma(a+k+\alpha+\beta+1) \Gamma(1+k)}{\Gamma(k+\beta+1) \Gamma(a+\alpha+\beta+1) \Gamma(a-k+1) \Gamma(k+1) \Gamma(1+k+v) \eta^{k}} x^{k+v} P_{\eta, b}^{(\alpha, \beta)}(y) .
\end{aligned}
$$

Approximating $x^{k+v} P_{\eta, b}^{(\alpha, \beta)}(y)$ by Jacobi polynomials in two variables, we obtain

$$
x^{k+v} P_{\eta, b}^{(\alpha, \beta)}(y) \approx \sum_{i=0}^{m} \sum_{j=0}^{m} S_{i, j, b} P_{\eta, i}^{(\alpha, \beta)}(x) P_{\eta, j}^{(\alpha, \beta)}(y),
$$

where $S_{i, j, b}=\left(\delta_{j, b} / R_{\eta, i}^{(\alpha, \beta)}, R_{\eta, j}^{(\alpha, \beta)}\right) \int_{0}^{\eta} \int_{0}^{\eta} x^{k+v} P_{\eta, b}^{(\alpha, \beta)}(y) P_{\eta, i}^{(\alpha, \beta)}(x) P_{\eta, j}^{(\alpha, \beta)}(y) W_{\eta}^{(\alpha, \beta)}(x, y) d x d y$. In view of the orthogonality relation, it becomes

$$
S_{i, j, b}=\frac{\delta_{j, b}}{R_{\eta, i}^{(\alpha, \beta)}} \int_{0}^{\eta} x^{k+v} P_{\eta, i}^{(\alpha, \beta)}(x) W_{\eta}^{(\alpha, \beta)}(x) d x .
$$

Or using (6) and (4),

$$
S_{i, j, b}=\frac{\delta_{j, b}}{R_{\eta, i}^{(\alpha, \beta)}} \sum_{l=0}^{i} \frac{(-1)^{i-l} \Gamma(i+\beta+1) \Gamma(i+l+\alpha+\beta+1)}{\Gamma(l+\beta+1) \Gamma(i+\alpha+\beta+1) \Gamma(i-l+1) \Gamma(l+1) \eta^{l}} \int_{0}^{\eta} x^{k+v+l+\beta}(\eta-x)^{\alpha} .
$$

The integrand in the above expression can be easily calculated by the well-known convolution theorem of the Laplace transformation

$$
£\left(\int_{0}^{\eta} x^{k+v+l+\beta}(\eta-x)^{\alpha}\right)=\frac{\Gamma(k+v+l+\beta+1) \Gamma(\alpha+1)}{s^{(k+v+l+\beta+\alpha+2)}} .
$$

Taking inverse Laplace,

$$
\int_{0}^{\eta} x^{k+v+l+\beta}(\eta-x)^{\alpha}=\frac{\Gamma(k+v+l+\beta+1) \Gamma(\alpha+1) \eta^{(k+v+l+\beta+\alpha+1)}}{\Gamma(k+v+l+\beta+\alpha+1)} .
$$


We can get the generalized coefficient of (20) as

$$
\begin{aligned}
S_{i j b}= & \frac{\delta_{j, b}}{R_{\eta, i}^{(\alpha, \beta)}} \sum_{l=0}^{i} \\
& \times \frac{(-1)^{i-l} \Gamma(i+\beta+1) \Gamma(i+l+\alpha+\beta+1) \Gamma(k+v+l+\beta+1) \Gamma(\alpha+1) \eta^{(k+v+l+\beta+\alpha+1)}}{\Gamma(l+\beta+1) \Gamma(i+\alpha+\beta+1) \Gamma(i-l+1) \Gamma(l+1) \eta^{l} \Gamma(k+v+l+\beta+\alpha+2)} .
\end{aligned}
$$

Using (7) and simplifying, we get the generalized value as

$$
\begin{aligned}
S_{i j b}= & \delta_{j, b} \sum_{l=0}^{i} \\
& \times \frac{(-1)^{i-l}(2 i+\alpha+\beta+1) \Gamma(i+1) \Gamma(i+l+\alpha+\beta+1) \Gamma(k+v+l+\beta+1) \Gamma(\alpha+1) \eta^{v}}{\Gamma(i+\alpha+1) \Gamma(l+\beta+1) \Gamma(i-l+1) \Gamma(l+1) \Gamma(k+v+l+\beta+\alpha+2)} .
\end{aligned}
$$

For simplicity of notation, we can write

$$
\Lambda_{a, k, v}=\frac{(-1)^{a-k} \Gamma(a+\beta+1) \Gamma(a+k+\alpha+\beta+1) \Gamma(1+k)}{\Gamma(k+\beta+1) \Gamma(a+\alpha+\beta+1) \Gamma(a-k+1) \Gamma(k+1) \Gamma(1+k+v) \eta^{k}} .
$$

Using (21), (22), (20) in (19), it follows that

$$
I_{x}^{v} P_{\eta, a}^{(\alpha, \beta)}(x) P_{\eta, b}^{(\alpha, \beta)}(y)=\sum_{k=0}^{a} \Lambda_{a, k, v} \sum_{i=0}^{m} \sum_{j=0}^{m} S_{i, j, b} P_{\eta, i}^{(\alpha, \beta)}(x) P_{\eta, j}^{(\alpha, \beta)}(y)
$$

Or

$$
I_{x}^{v} P_{\eta, a}^{(\alpha, \beta)}(x) P_{\eta, b}^{(\alpha, \beta)}(y)=\sum_{i=0}^{m} \sum_{j=0}^{m} \sum_{k=0}^{a} \Lambda_{a, k, v} S_{i, j, b} P_{\eta, i}^{(\alpha, \beta)}(x) P_{\eta, j}^{(\alpha, \beta)}(y) .
$$

Let

$$
\Theta_{i, j, a, b, k}=\sum_{k=0}^{a} \Lambda_{a, k, v} S_{i, j, b}
$$

Then

$$
I_{x}^{v} P_{\eta, a}^{(\alpha, \beta)}(x) P_{\eta, b}^{(\alpha, \beta)}(y)=\sum_{i=0}^{m} \sum_{j=0}^{m} \Theta_{i, j, a, b, k} P_{\eta, i}^{(\alpha, \beta)}(x) P_{\eta, j}^{(\alpha, \beta)}(y) .
$$

Using the notation $r=M i+j+1, q=M a+b+1, \Omega_{q, r, k}=\Theta_{i, j, b, a, k}$ for $i, j, a, b=$ $0,1,2,3, \ldots, m$, we get the desired result.

Lemma 3.2. Let $\Psi_{M^{2}}(x, y)$ be as defined in (15). Then the derivative of order $\sigma$ of $\Psi_{M^{2}}(x, y)$ with respect to $y$ is given by

$$
D_{y}^{\sigma}\left(\Psi_{M^{2}}(x, y)\right) \simeq H_{M^{2} \times M^{2}}^{\sigma, y} \Psi_{M^{2}}(x, y)
$$


where $H_{M^{2} \times M^{2}}^{\sigma, y}$ is the operational matrix of derivative of order $\sigma$ and is defined as

$$
H_{M^{2} \times M^{2}}^{\sigma, y}=\left(\begin{array}{cccccc}
\Omega_{1,1, k} & \Omega_{1,2, k} & \cdots & \Omega_{1, r, k} & \cdots & \Omega_{1, M^{2}, k} \\
\Omega_{2,1, k} & \Omega_{2,2, k} & \cdots & \Omega_{2, r, k} & \cdots & \Omega_{2, M^{2}, k} \\
\vdots & \vdots & \vdots & \vdots & \vdots & \vdots \\
\Omega_{q, 1, k} & \Omega_{q, 2, k} & \cdots & \Omega_{q, r, k} & \cdots & \Omega_{q, M^{2}, k} \\
\vdots & \vdots & \vdots & \vdots & \vdots & \vdots \\
\Omega_{M^{2}, 1, k} & \Omega_{M^{2}, 2, k} & \cdots & \Omega_{M^{2}, r, k} & \cdots & \Omega_{M^{2}, M^{2}, k}
\end{array}\right)
$$

and $q=M i+j+1, r=M a+b+1, \Omega_{q, r, k}=\Theta_{i, j, a, b, k}$ for $i, j, a, b=0,1,2, \ldots, m$,

$$
\Theta_{i, j, a, b, k}=\sum_{k=0}^{a} \Lambda_{a, k, \sigma} S_{i, j, b}
$$

$$
\begin{aligned}
S_{i j b}= & \delta_{j, b} \sum_{l=0}^{i} \\
& \times \frac{(-1)^{i-l}(2 i+\alpha+\beta+1) \Gamma(i+1) \Gamma(i+l+\alpha+\beta+1) \Gamma(k-\sigma+l+\beta+1) \Gamma(\alpha+1) \eta^{\sigma}}{\Gamma(i+\alpha+1) \Gamma(l+\beta+1) \Gamma(i-l+1) \Gamma(l+1) \Gamma(k-\sigma+l+\beta+\alpha+2)},
\end{aligned}
$$

and

$$
\Lambda_{a, k, \sigma}=\frac{(-1)^{a-k} \Gamma(a+\beta+1) \Gamma(a+k+\alpha+\beta+1) \Gamma(1+k)}{\Gamma(k+\beta+1) \Gamma(a+\alpha+\beta+1) \Gamma(a-k+1) \Gamma(k+1) \Gamma(1+k-\sigma) \eta^{k}} .
$$

Proof. The proof of this Lemma is similar to the proof of Lemma 3.1.

Lemma 3.3. Let $\Psi_{M^{2}}(x, y)$ be as defined in (15). Then the derivative of order $\sigma$ of $\Psi_{M^{2}}(x, y)$ with respect to $x$ is given by

$$
D_{x}^{\sigma}\left(\Psi_{M^{2}}(x, y)\right) \simeq H_{M^{2} \times M^{2}}^{\sigma, x} \Psi_{M^{2}}(x, y)
$$

where $H_{M^{2} \times M^{2}}^{\sigma, x}$ is the operational matrix of derivative of order $\sigma$ with respect to $\mathrm{x}$ and is defined as

$$
H_{M^{2} \times M^{2}}^{\sigma, x}=\left(\begin{array}{cccccc}
\Omega_{1,1, k} & \Omega_{1,2, k} & \cdots & \Omega_{1, r, k} & \cdots & \Omega_{1, M^{2}, k} \\
\Omega_{2,1, k} & \Omega_{2,2, k} & \cdots & \Omega_{2, r, k} & \cdots & \Omega_{2, M^{2}, k} \\
\vdots & \vdots & \vdots & \vdots & \vdots & \vdots \\
\Omega_{q, 1, k} & \Omega_{q, 2, k} & \cdots & \Omega_{q, r, k} & \cdots & \Omega_{q, M^{2}, k} \\
\vdots & \vdots & \vdots & \vdots & \vdots & \vdots \\
\Omega_{M^{2}, 1, k} & \Omega_{M^{2}, 2, k} & \cdots & \Omega_{M^{2}, r, k} & \cdots & \Omega_{M^{2}, M^{2}, k}
\end{array}\right)
$$

and $r=M i+j+1, q=M a+b+1, \Omega_{q, r, k}=\Theta_{i, j, a, b, k}$ for $i, j, a, b=0,1,2, \ldots, m$,

$$
\Theta_{i, j, a, b, k}=\sum_{k=0}^{a} \Lambda_{a, k, \sigma} S_{i, j, b}
$$




$$
\begin{aligned}
S_{i j b}= & \delta_{j, b} \sum_{l=0}^{i} \\
& \times \frac{(-1)^{i-l}(2 i+\alpha+\beta+1) \Gamma(i+1) \Gamma(i+l+\alpha+\beta+1) \Gamma(k-\sigma+l+\beta+1) \Gamma(\alpha+1) \eta^{\sigma}}{\Gamma(i+\alpha+1) \Gamma(l+\beta+1)(i-l) ! l ! \Gamma(k-\sigma+l+\beta+\alpha+2)},
\end{aligned}
$$

and

$$
\Lambda_{a, k, \sigma}=\frac{(-1)^{a-k} \Gamma(a+\beta+1) \Gamma(a+k+\alpha+\beta+1) \Gamma(1+k)}{\Gamma(k+\beta+1) \Gamma(a+\alpha+\beta+1) \Gamma(a-k+1) \Gamma(k+1) \Gamma(1+k-\sigma) \eta^{k}} .
$$

Proof. The proof of this Lemma is similar to the proof of Lemma 3.1.

REMARK 1. Note that if $f=L_{M^{2}} \Psi(x, y)$, then, for all $c_{i} \in R$,

$$
\sum_{i=0}^{n} c_{i} \frac{\partial^{i} f}{\partial y^{i}}=L_{M^{2}} H_{c_{i}}^{y} \Psi(x, y)
$$

where

$$
H_{c_{i}}^{y}=\sum_{i=0}^{n} c_{i} H_{M^{2} \times M^{2}}^{i, y}
$$

Proof. The proof of this remark is straightforward.

\section{Main result}

Application of the operational matrices to coupled systems of fractional-order PDEs

In this section, we are interested in the approximate solution of a generalized class of coupled systems of fractional-order PDEs of the form

$$
\begin{aligned}
& \frac{\partial^{\alpha} U}{\partial x^{\alpha}}=\sum_{i=0}^{n} a_{i} \frac{\partial^{i} U}{\partial y^{i}}+\sum_{i=0}^{n} b_{i} \frac{\partial^{i} V}{\partial x^{i}}+\sum_{i=0}^{n} c_{i} \frac{\partial^{i} V}{\partial y^{i}}+F_{1}, \\
& \frac{\partial^{\alpha} V}{\partial x^{\alpha}}=\sum_{i=0}^{n} d_{i} \frac{\partial^{i} V}{\partial y^{i}}+\sum_{i=0}^{n} e_{i} \frac{\partial^{i} U}{\partial x^{i}}+\sum_{i=0}^{n} f_{i} \frac{\partial^{i} U}{\partial y^{i}}+F_{2},
\end{aligned}
$$

subject to the initial conditions

$$
U^{(i)}(0, y)=H_{i}(y), \quad V^{(i)}(0, y)=G_{i}(y) \quad \text { for } i=0,1, \ldots, n .
$$

We seek the solution of the above problem in terms of shifted Jacobi polynomials of order $M$ in the matrix form such that

$$
\frac{\partial^{\sigma} U(x, y)}{\partial x^{\sigma}}=K_{M^{2}} \Psi(x, y), \quad \frac{\partial^{\sigma} V(x, y)}{\partial x^{\sigma}}=L_{M^{2}} \Psi(x, y) .
$$

Applying integration of order $\sigma$ with respect to $x$ and using the initial conditions as defined in $(40)$,

$$
U(x, y)=K_{M^{2}} P_{M^{2} \times M^{2}}^{\alpha} \Psi(x, y)+\sum_{i=0}^{n} x^{i} H_{i}(y), \quad V(x, y)=L_{M^{2}} P_{M^{2} \times M^{2}}^{\beta} \Psi(x, y)+\sum_{i=0}^{n} x^{i} G_{i}(y) .
$$


Approximating $\sum_{i=0}^{n} x^{i} H_{i}(y)$ and $\sum_{i=0}^{n} x^{i} G_{i}(y)$ with two-dimensional Jacobi polynomials, we can write

$$
\sum_{i=0}^{n} x^{i} H_{i}(y)=F^{H} \Psi(x, y), \quad \sum_{i=0}^{n} x^{i} G_{i}(y)=F^{G} \Psi(x, y) .
$$

Substituting in (42),

$$
U(x, y)=\left(K_{M^{2}} P_{M^{2} \times M^{2}}^{\sigma}+F^{H}\right) \Psi(x, y), \quad V(x, y)=\left(L_{M^{2}} P_{M^{2} \times M^{2}}^{\sigma}+F^{G}\right) \Psi(x, y) .
$$

For simplicity of notation, let

$$
\hat{K}=\left(K_{M^{2}} P_{M^{2} \times M^{2}}^{\sigma}+F^{H}\right), \quad \hat{L}=\left(L_{M^{2}} P_{M^{2} \times M^{2}}^{\sigma}+F^{G}\right) .
$$

Using the simplified notation,

$$
U(x, y)=\hat{K} \Psi(x, y), \quad V(x, y)=\hat{L} \Psi(x, y) .
$$

Now, using (37) and (38),

$$
\begin{aligned}
& \sum_{i=0}^{n} a_{i} \frac{\partial^{i} U}{\partial y^{i}}=\hat{K} H_{a_{i}}^{y} \Psi(x, y), \quad \sum_{i=0}^{n} b_{i} \frac{\partial^{i} V}{\partial x^{i}}=\hat{L} H_{b_{i}}^{x} \Psi(x, y), \\
& \sum_{i=0}^{n} c_{i} \frac{\partial^{i} V}{\partial y^{i}}=\hat{L} H_{c_{i}}^{y} \Psi(x, y), \quad \sum_{i=0}^{n} d_{i} \frac{\partial^{i} U}{\partial y^{i}}=\hat{K} H_{d_{i}}^{y} \Psi(x, y), \\
& \sum_{i=0}^{n} e_{i} \frac{\partial^{i} V}{\partial x^{i}}=\hat{L} H_{e_{i}}^{x} \Psi(x, y), \quad \sum_{i=0}^{n} f_{i} \frac{\partial^{i} V}{\partial y^{i}}=\hat{L} H_{f_{i}}^{y} \Psi(x, y) .
\end{aligned}
$$

We write the source terms in matrix form as $F_{1}=\hat{F}_{1} \Psi(x, y)$ and $F_{2}=\hat{F}_{2} \Psi(x, y)$. Using (41) and (46) in (39),

$$
\begin{aligned}
& K_{M^{2}} \Psi(x, y)=\hat{K} H_{a_{i}}^{y} \Psi(x, y)+\hat{L} H_{b_{i}}^{x} \Psi(x, y)+\hat{L} H_{c_{i}}^{y} \Psi(x, y)+\hat{F}_{1} \Psi(x, y), \\
& L_{M^{2}} \Psi(x, y)=\hat{L} H_{d_{i}}^{y} \Psi(x, y)+\hat{K} H_{e_{i}}^{x} \Psi(x, y)+\hat{K} H_{f_{i}}^{y} \Psi(x, y)+\hat{F}_{2} \Psi(x, y) .
\end{aligned}
$$

This can be rewritten in matrix form as

$$
\begin{aligned}
\left(\begin{array}{c}
K_{M^{2}}^{T} \Psi(x, y) \\
L_{M^{2}}^{T} \Psi(x, y)
\end{array}\right)= & \left(\begin{array}{c}
\hat{K} H_{a_{i}}^{y} \Psi(x, y) \\
\hat{L} H_{d_{i}}^{y} \Psi(x, y)
\end{array}\right)+\left(\begin{array}{c}
\hat{L} H_{b_{i}}^{x} \Psi(x, y) \\
\hat{K} H_{e_{i}}^{x} \Psi(x, y)
\end{array}\right) \\
& +\left(\begin{array}{c}
\hat{L} H_{c_{i}}^{y} \Psi(x, y) \\
\hat{K} H_{f_{i}}^{y} \Psi(x, y)
\end{array}\right)+\left(\begin{array}{l}
\hat{F}_{1} \Psi(x, y) \\
\hat{F}_{2} \Psi(x, y)
\end{array}\right)
\end{aligned}
$$

Taking the transpose of the system and simplifying,

$$
\begin{aligned}
\left(\begin{array}{cc}
K_{M^{2}}^{T} & L_{M^{2}}^{T}
\end{array}\right) A= & \left(\begin{array}{ll}
\hat{K} H_{a_{i}}^{y} & \hat{L} H_{d_{i}}^{y}
\end{array}\right) A+\left(\begin{array}{ll}
\hat{L} H_{b_{i}}^{x} & \hat{K} H_{e_{i}}^{x}
\end{array}\right) A+\left(\begin{array}{ll}
\hat{L} H_{c_{i}}^{y} & \hat{K} H_{f_{i}}^{y}
\end{array}\right) A \\
& +\left(\begin{array}{ll}
\hat{F}_{1} & \hat{F}_{2}
\end{array}\right) A
\end{aligned}
$$

where

$$
A=\left(\begin{array}{cc}
\Psi(x, y) & O_{M} \\
O_{M} & \Psi(x, y)
\end{array}\right)
$$


We can also write the system as

$$
\begin{gathered}
\left(\begin{array}{ll}
K_{M^{2}}^{T} & L_{M^{2}}^{T}
\end{array}\right)-\left(\begin{array}{ll}
\hat{K} H_{a_{i}}^{y} & \hat{L} H_{d_{i}}^{y}
\end{array}\right)-\left(\begin{array}{ll}
\hat{L} H_{b_{i}}^{x} & \hat{K} H_{e_{i}}^{x}
\end{array}\right)-\left(\begin{array}{ll}
\hat{L} H_{c_{i}}^{y} & \hat{K} H_{f_{i}}^{y}
\end{array}\right) \\
-\left(\begin{array}{ll}
\hat{F_{1}} & \hat{F}_{2}
\end{array}\right)=0 \\
\left(\begin{array}{ll}
K_{M^{2}}^{T} & L_{M^{2}}^{T}
\end{array}\right)-\left(\begin{array}{cc}
\hat{K} & \hat{L}
\end{array}\right)\left(\begin{array}{cc}
H_{a_{i}}^{y} & O_{M^{2} \times M^{2}} \\
O_{M^{2} \times M^{2}} & H_{d_{i}}^{y}
\end{array}\right)-\left(\begin{array}{ll}
\hat{K} & \hat{L}
\end{array}\right)\left(\begin{array}{cc}
O_{M^{2} \times M^{2}} & H_{e_{i}}^{x} \\
H_{b_{i}}^{x} & O_{M^{2} \times M^{2}}
\end{array}\right) \\
-\left(\begin{array}{cc}
\hat{K} & \hat{L}
\end{array}\right)\left(\begin{array}{cc}
O_{M^{2} \times M^{2}} & H_{f_{i}}^{y} \\
H_{c_{i}}^{y} & O_{M^{2} \times M^{2}}
\end{array}\right)-\left(\begin{array}{ll}
\hat{F}_{1} & \hat{F}_{2}
\end{array}\right)=0, \\
\\
\Rightarrow\left(\begin{array}{ll}
K_{M^{2}}^{T} & L_{M^{2}}^{T}
\end{array}\right)-\left(\begin{array}{cc}
\hat{K} & \hat{L}
\end{array}\right)\left(\begin{array}{cc}
H_{a_{i}}^{y} & H_{e_{i}}^{x}+H_{f_{i}}^{y} \\
H_{c_{i}}^{y}+H_{b_{i}}^{x} & H_{d_{i}}^{y}
\end{array}\right)-\left(\begin{array}{ll}
\hat{F}_{1} & \hat{F}_{2}
\end{array}\right)=0 .
\end{gathered}
$$

Using (44) and after a small modification,

$$
\begin{aligned}
\Rightarrow & \left(\begin{array}{ll}
K_{M^{2}}^{T} & L_{M^{2}}^{T}
\end{array}\right)-\left(\begin{array}{ll}
K_{M^{2}} P_{M^{2} \times M^{2}}^{\sigma} & L_{M^{2}} P_{M^{2} \times M^{2}}^{\sigma}
\end{array}\right)\left(\begin{array}{cc}
H_{a_{i}}^{y} & H_{e_{i}}^{x}+H_{f_{i}}^{y} \\
H_{c_{i}}^{y}+H_{b_{i}}^{x} & H_{d_{i}}^{y}
\end{array}\right) \\
& -\left(\begin{array}{cc}
F^{H} & F^{G}
\end{array}\right)\left(\begin{array}{cc}
H_{a_{i}}^{y} & H_{e_{i}}^{x}+H_{f_{i}}^{y} \\
H_{c_{i}}^{y}+H_{b_{i}}^{x} & H_{d_{i}}^{y}
\end{array}\right)-\left(\begin{array}{ll}
\hat{F}_{1} & \hat{F}_{2}
\end{array}\right)=0 .
\end{aligned}
$$

This can be written in the form

$$
X-X B-C=0
$$

where

$$
\begin{aligned}
& X=\left(\begin{array}{ll}
K_{M^{2}}^{T} & L_{M^{2}}^{T}
\end{array}\right), \\
& B=\left(\begin{array}{cc}
P_{M^{2} \times M^{2}}^{\sigma}\left(H_{a_{i}}^{y}\right) & P_{M^{2} \times M^{2}}^{\sigma}\left(H_{e_{i}}^{x}+H_{f_{i}}^{y}\right) \\
P_{M^{2} \times M^{2}}^{\sigma}\left(H_{c_{i}}^{y}+H_{b_{i}}^{x}\right) & P_{M^{2} \times M^{2}}^{\sigma}\left(H_{d_{i}}^{y}\right)
\end{array}\right)
\end{aligned}
$$

and

$$
C=\left(\begin{array}{ll}
F^{H} & F^{G}
\end{array}\right)\left(\begin{array}{cc}
H_{a_{i}}^{y} & H_{e_{i}}^{x}+H_{f_{i}}^{y} \\
H_{c_{i}}^{y}+H_{b_{i}}^{x} & H_{d_{i}}^{y}
\end{array}\right)+\left(\begin{array}{ll}
\hat{F}_{1} & \hat{F}_{2}
\end{array}\right),
$$

which is a system of algebraic equations. Note that the system is of the form of a Lyapunov matrix equation, and thus finding its solution without using software is not easy. We use Matlab for numerical simulations. By solving (49), we can get $K_{M^{2}}^{T}$ and $L_{M^{2}}^{T}$, which can be used in (43) to get the approximate solution.

\section{Illustrative examples}

We analyze the new algorithm with three test examples. The first example is analyzed by using the parameters $\alpha=1, \beta=1$, while in the second example, we use the values $\alpha=2, \beta=2$. In both cases, we observe a high accuracy. Different error norms are used to analyze the accuracy 
and efficiency of proposed method. Also note that all the experiments are analyzed using the domain $[0, \eta]=[0,1]$. In order to understand the efficiency of the scheme, we display the results graphically. In last example, we compare the proposed method with that of the differential transform method. The simulation was carried out by using MatLab commands in my personal computer with $2.5 \mathrm{GHz}$ processor.

EXAMPLE 5.1. Consider the system of two general coupled arbitrary-order non-homogeneous PDEs as classified in $[\mathbf{1 1}]$, in the form

$$
\begin{aligned}
& \frac{\partial^{\sigma} U(x, y)}{\partial x^{\sigma}}=2 \frac{\partial^{2} U(x, y)}{\partial y^{2}}+3 \frac{\partial^{2} V(x, y)}{\partial x^{2}}+\frac{\partial V(x, y)}{\partial y}+F_{1}(x, y) \\
& \frac{\partial^{\sigma} V(x, y)}{\partial x^{\alpha}}=3 \frac{\partial^{2} V(x, y)}{\partial y^{2}}+4 \frac{\partial^{2} U(x, y)}{\partial x^{2}}+\frac{\partial U(x, y)}{\partial y}+F_{2}(x, y)
\end{aligned}
$$

subject to the initial conditions $U^{(i)}(0, y)=0, V^{(i)}(0, y)=0, i=0,1,2$,

where $2<\sigma \leqslant 3$ and the external or source terms are given by

$$
\begin{aligned}
& F_{1}(x, y)=-\frac{x\left(36 x-24 e^{2 y}+2 x^{3} e^{2 y}-x^{3}\right)}{e^{y}}, \\
& F_{2}(x, y)=-\frac{x\left(48 x e^{2 y}+x^{3} e^{2 y}+3 x^{3}-24\right)}{e^{y}} .
\end{aligned}
$$

The exact solution at $\sigma=3$ is known and is given as

$$
U(x, y)=e^{y} x^{4}, \quad V(x, y)=e^{-y} x^{4} .
$$

We approximate the solution of the above problem with different scale levels and observe that the method is very efficient and provides a highly accurate estimate of the solutions. We observe that the result is very accurate even for very a small level of $M$. Comparison of the exact $U(x, y)$ and $V(x, y)$ with the approximate solutions obtained with this new technique for scale level $M=6$ is shown in Figure 1. One can easily see that the approximate solutions approach the exact solutions at classical order very well. In order to analyze the absolute error of approximation, we approximate the solutions at different scale levels, using $M=5,6,7$, and observe that the absolute amount of error decreases significantly with an increase of scale level $M$ (see Figure 2). This property also demonstrates the validity of (16). From Figure 2, we can easily observe that, at scale level $M=7$, the absolute error is much less than $10^{-5}$, which is an acceptable number for such complicated problems. We approximate the solution of the scheme at some fractional value of $\sigma$ and observe that, as $\sigma \rightarrow 3$, the result approaches the exact solution. This phenomenon is visualized in Figure 3. Note that, for this problem, we use the parameters as $\alpha=1$ and $\beta=1$.

EXAMPLE 5.2. Consider the coupled system of multi-term non-homogenous fractionalorder PDEs which is the generalization of some system of classical PDEs as discussed in $[\mathbf{1 1}]$, given by

$$
\begin{aligned}
& \frac{\partial^{\sigma} U(x, y)}{\partial x^{\sigma}}=6 \frac{\partial^{2} U(x, y)}{\partial y^{2}}+5 \frac{\partial V(x, y)}{\partial y}+F_{1}(x, y) \\
& \frac{\partial^{\sigma} V(x, y)}{\partial x^{\sigma}}=3 \frac{\partial^{2} V(x, y)}{\partial y^{2}}+4 \frac{\partial^{2} U(x, y)}{\partial x^{2}}+5 \frac{\partial U(x, y)}{\partial y}+F_{2}(x, y)
\end{aligned}
$$

subject to the initial conditions $U^{(i)}(0, y)=0, V^{(i)}(0, y)=0, i=0,1,2$, 


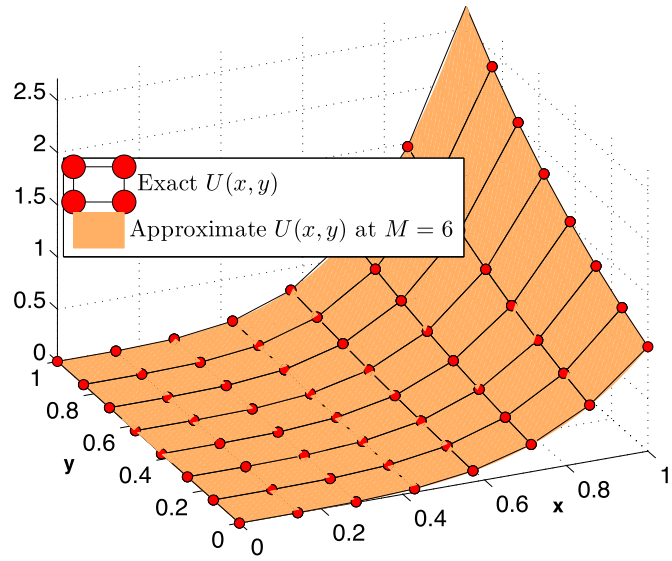

(a)

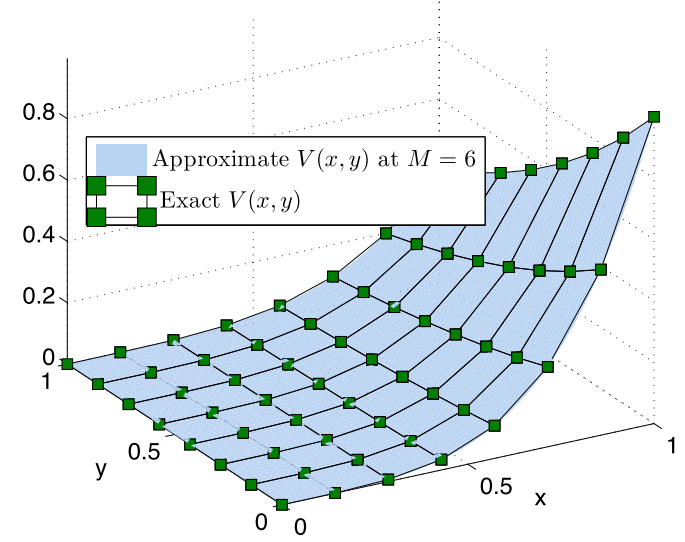

(b)

Figure 1. Comparison of approximate and exact solution $U(x, y)(\mathrm{a})$ and $V(x, y)(\mathrm{b})$ of Example (5.1). Red dots and green squares represents exact $U(x, y)$ and $V(x, y)$, while the surfaces represent approximate solutions, respectively. Here we set parameters $\alpha=1, \beta=1, M=6$ and $\sigma=3$.

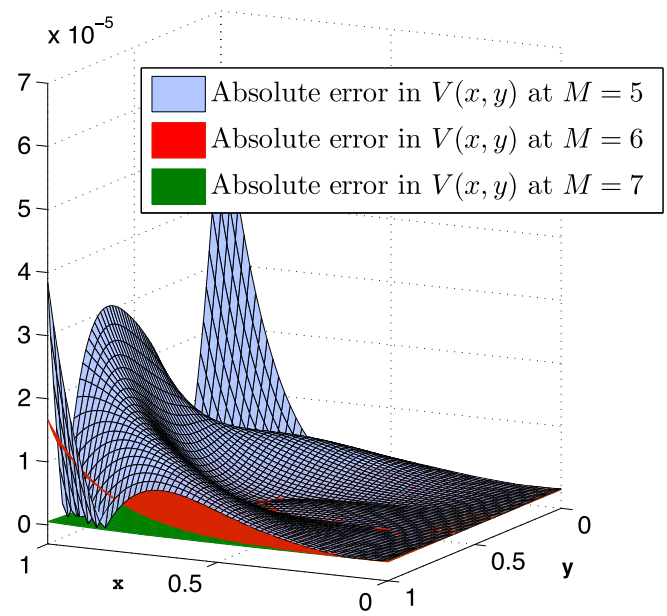

(a)

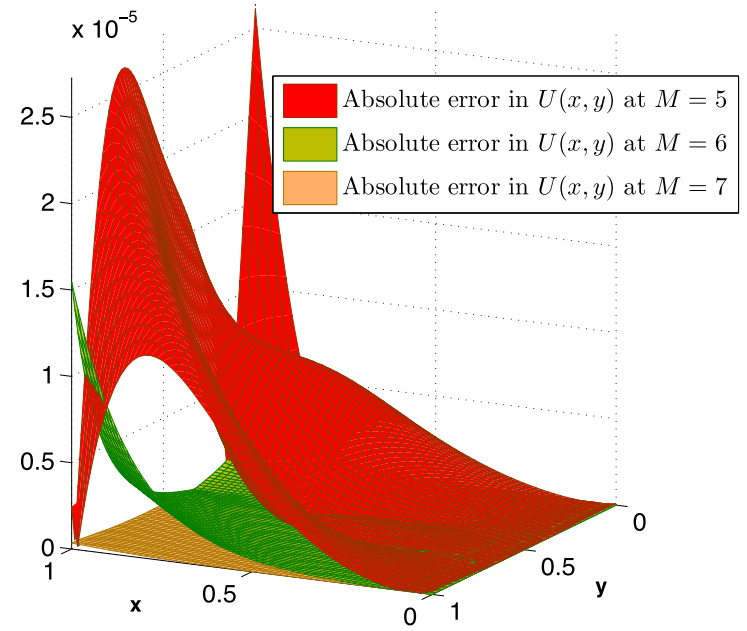

(b)

Figure 2. Absolute error in $U(x, y)$ (a) and $V(x, y)$ (b) of Example (5.1) at different scale levels ranging from $M=5$ to $M=7$. Here we use the parameters $\alpha=1, \beta=1$ and $\sigma=3$.

where the source terms $F_{1}(x, y)$ and $F_{2}(x, y)$ are defined as

$$
F_{1}(x, y)=-60 x^{4} y^{3}-144 x^{4} y^{2}-30 x^{3} y^{2}+108 x^{3} y+48 x y^{4}-18 y^{3}
$$

and

$$
F_{2}(x, y)=-40 x^{4} y^{3}-108 x^{4} y^{2}+45 x^{3} y^{2}-36 x^{3} y-96 x^{2} y^{4}+72 x y^{4}+72 x y^{3}+12 y^{3} .
$$

The exact solution of the above problem for $\sigma=3$ is given as

$$
U(x, y)=2 x^{4} y^{4}-3 x^{3} y^{3}
$$




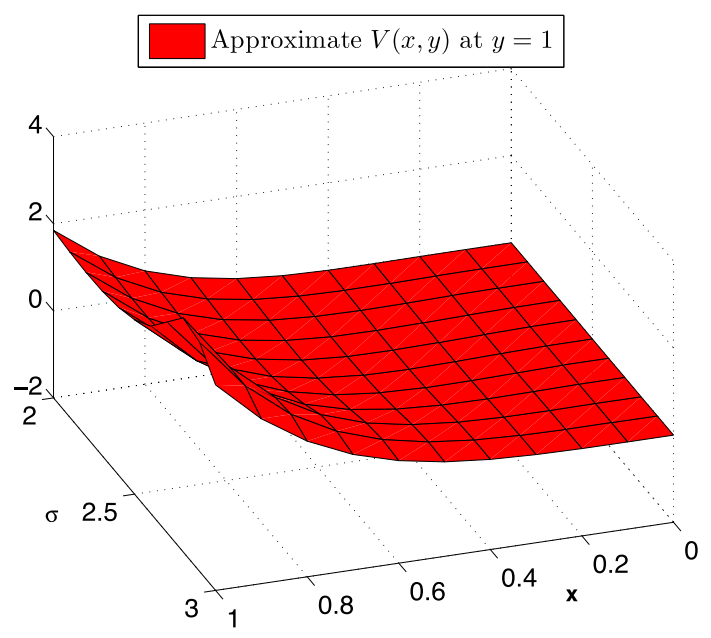

(a)

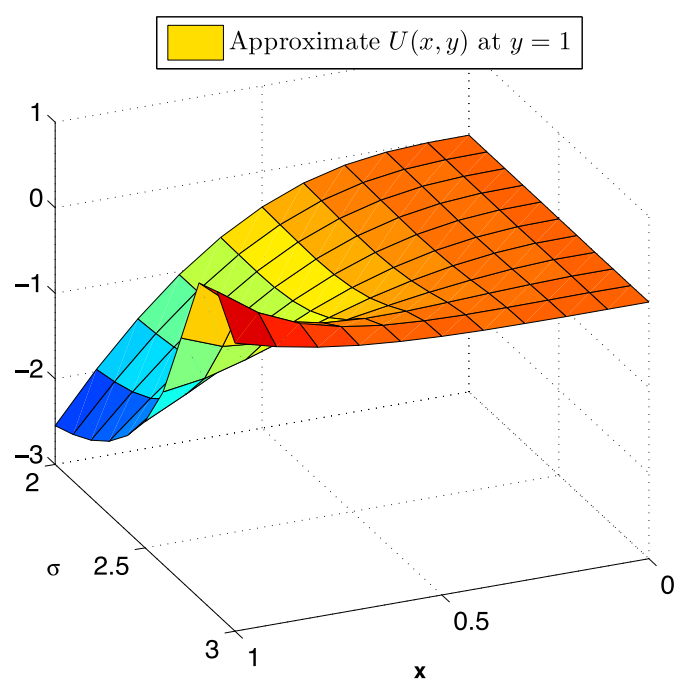

(b)

Figure 3. (a) Approximate $V(x, y)$ at $y=1$ and at fractional values of $\sigma, 2<\sigma \leqslant 3$, of Example (5.1). Setting $\alpha=1, \beta=1, M=6$. (b) Approximate $U(x, y)$ at $y=1$ and at fractional values of $\sigma, 2<\sigma \leqslant 3$. Setting $\alpha=1, \beta=1, M=6$.

and

$$
V(x, y)=3 x^{4} y^{4}+2 x^{3} y^{3}
$$

We approximate the solution of the problem with the proposed method and, as expected, we get a highly accurate solution. We found that, at small scale level $M=6$, the method provides a highly accurate solution. The comparison of the approximate solution with the exact solution is displayed in Figure 4, where one can easily see that the approximate solution coincides with the exact solution. We approximate the solution of the above problem for fractional values of $\sigma$ and the same conclusion is made about the behavior of the solution. The numerical solution approaches uniformly to the exact solution at $\sigma=3$ as $\sigma \rightarrow 3$. The results for fractional values of $\sigma$ and at $y=0.5$ are displayed in Figure 5 and the results at $y=0.8$ are displayed in Figure 6. This phenomenon is one of the basic properties of fractional calculus, that is, as the order approaches from a fractional to an integer value, the solution approaches the specified solution at integer order. This shows that the method provides the best approximate solution for fractional values of $\sigma$.

EXAMPLE 5.3. Following $[\mathbf{3}, \mathbf{3 6}, \mathbf{5 8}]$, we consider the coupled system of fractional-order PDEs given by

$$
\begin{aligned}
& \frac{\partial^{\sigma} U(x, y)}{\partial x^{\sigma}}=\frac{\partial^{\sigma} U(x, y)}{\partial y^{\sigma}}+2 V(x, y), \\
& \frac{\partial^{\sigma} V(x, y)}{\partial x^{\sigma}}=-\frac{\partial^{\sigma} U(x, y)}{\partial y^{\sigma}}-2 U(x, y),
\end{aligned}
$$




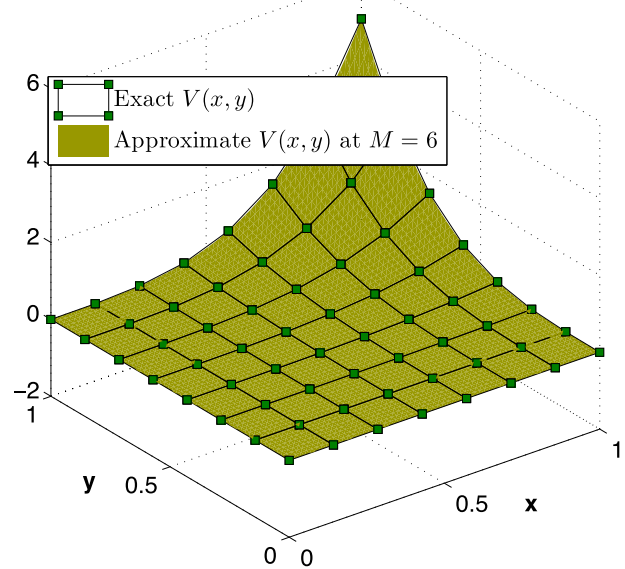

(a)

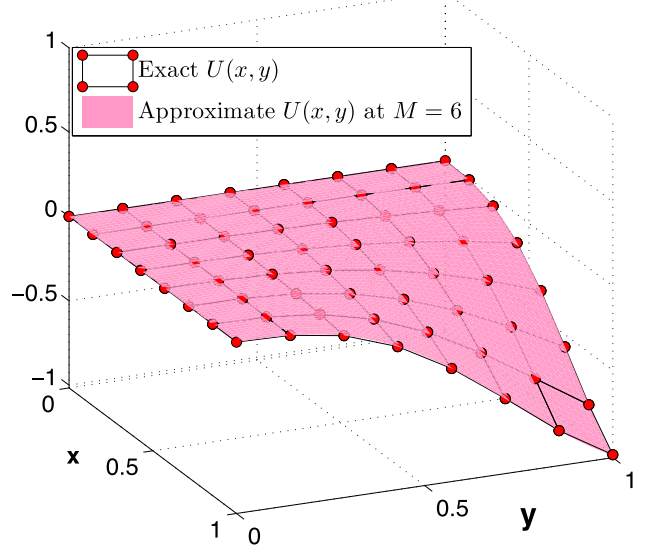

(b)

Figure 4. Comparison of approximate and exact solution $U(x, y)$ (a) and $V(x, y)$ (b) of Example (5.2). Red dots and green squares represents exact $U(x, y)$ and $V(x, y)$ while the surfaces represents approximate solutions, respectively. Here we set parameters $\alpha=2, \beta=2, M=6$ and $\sigma=3$.

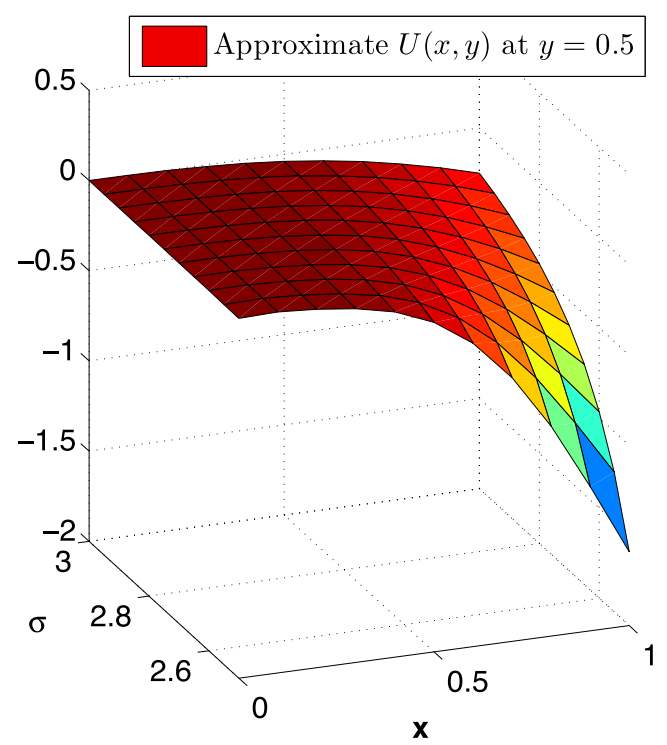

(a)

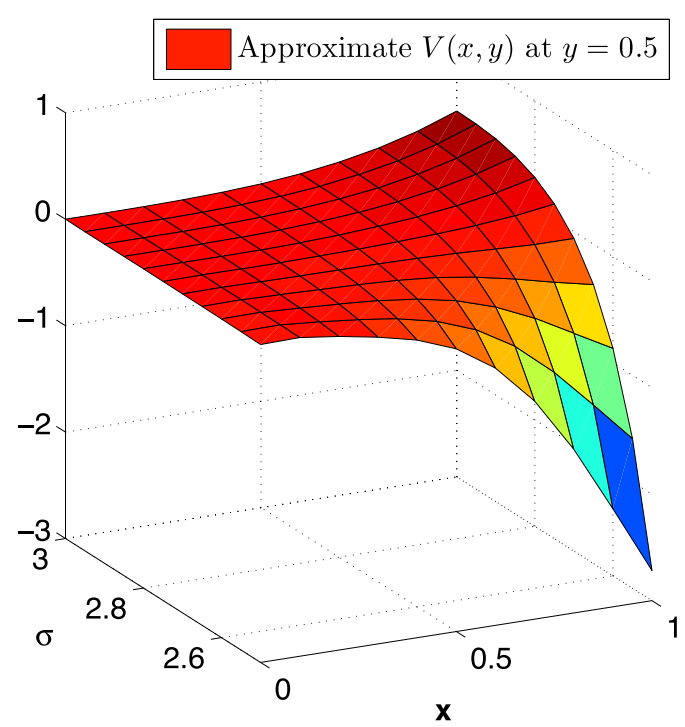

(b)

Figure 5. (a) Approximate $U(x, y)$ at $y=0.5$ and at fractional values of $\sigma, 2.5<\sigma \leqslant 3$, of Example (5.2). (b) Approximate $V(x, y)$ at $y=0.5$ and at fractional values of $\sigma, 2.5<\sigma \leqslant 3$. Setting $\alpha=2, \beta=2$ and $M=6$ of Example (5.2).

subject to the initial conditions

$$
U(0, y)=\sin (y) \quad \text { and } \quad V(0, y)=\cos (y)
$$

The exact solution of the problem at $\sigma=1$ is $U(x, y)=\sin (x+y)$ and $V(x, y)=\cos (x+y)$. In $[3,58]$, the differential transform method is employed to solve this problem. We apply 


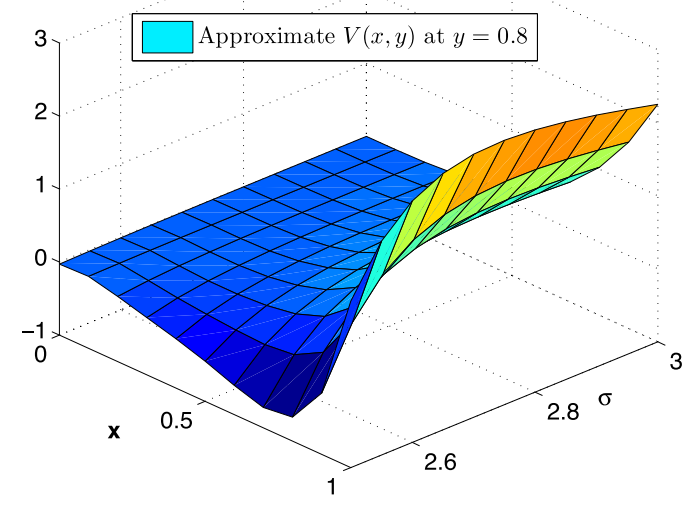

(a)

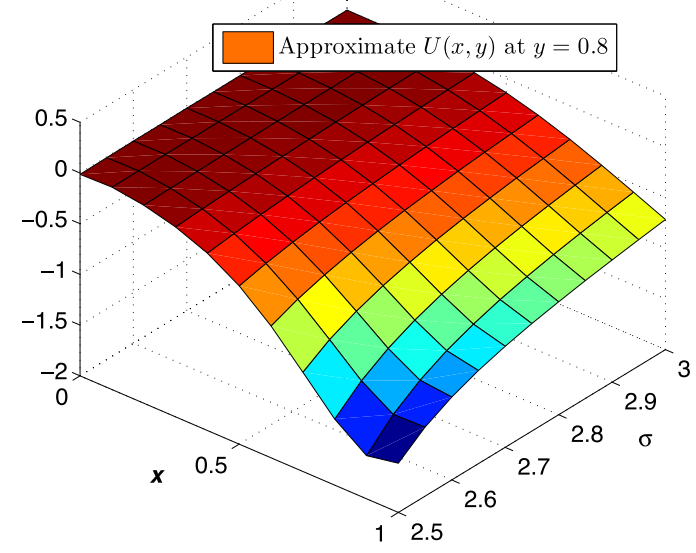

(b)

Figure 6. (a) Approximate $U(x, y)$ at $y=0.8$ and at fractional values of $\sigma, 2.5<\sigma \leqslant 3$, of Example (5.2). (b) Approximate $V(x, y)$ at $y=0.8$ and at fractional values of $\sigma, 2.5<\sigma \leqslant 3$. Setting $\alpha=2, \beta=2$ and $M=6$ of Example (5.2).

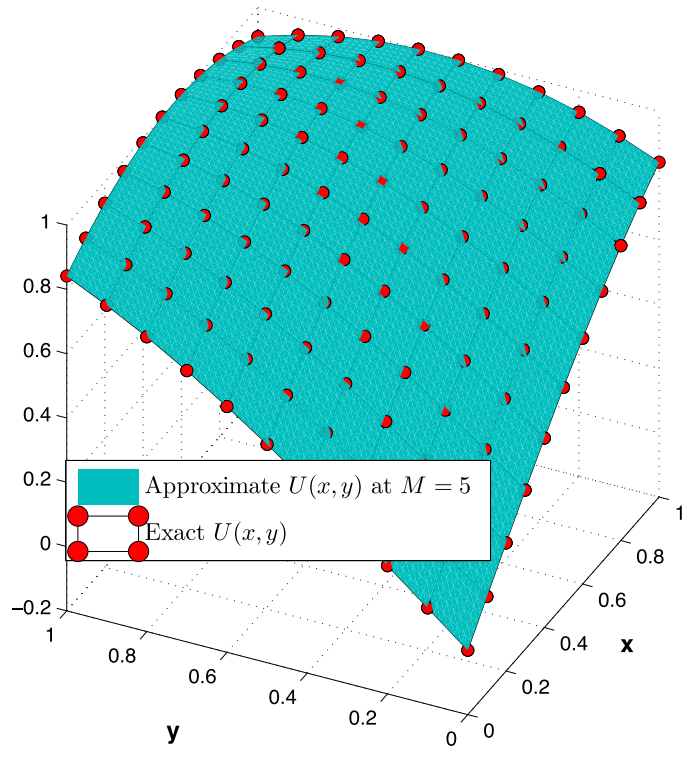

(a)

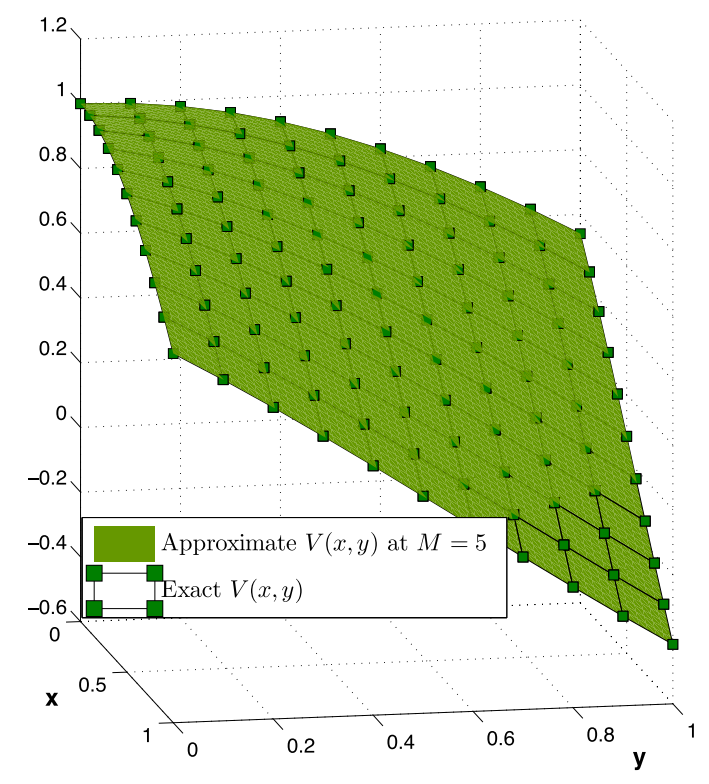

(b)

Figure 7. (a) Approximate $U(x, y)$ at $y=0.8$ and at fractional values of $\sigma, 1 \leqslant \sigma<2$, of Example (5.3). (b) Approximate $V(x, y)$ at $y=0.8$ and at fractional values of $\sigma, 1 \leqslant \sigma<2$. Setting $\alpha=2, \beta=2, \sigma=1$ and $M=6$.

the presented algorithm and observe that the solution is highly accurate. The comparison of the exact and the approximate solution for $M=5$ is shown in Figure 7. One can easily note that the solution matches very well. In order to compare the accuracy of the proposed 
method against other methods in the literature, we compare the absolute error with some results reported in [36]. These results are displayed in Table 1.

TABle 1. Comparison of absolute error in the exact and the approximate solutions obtained with the proposed method and the error reported in [36] for Example (5.3).

\begin{tabular}{clclccc}
\hline$x \backslash y=0.1$ & $U_{L}^{e}[\mathbf{3 6}]$ & $U_{C}^{e}[\mathbf{3 6}]$ & \multicolumn{1}{c}{$U_{P}^{e}$} & $V_{L}^{e}[\mathbf{3 6}]$ & $V_{C}^{e}[\mathbf{3 6}]$ & $V_{P}^{e}$ \\
\hline 0.0 & $3.6\left(10^{-5}\right)$ & $6.0\left(10^{-5}\right)$ & $4.04\left(10^{-8}\right)$ & $3.9\left(10^{-5}\right)$ & $6.0\left(10^{-5}\right)$ & $5.4\left(10^{-8}\right)$ \\
0.1 & $1.1\left(10^{-4}\right)$ & $3.1\left(10^{-5}\right)$ & $1.8\left(10^{-9}\right)$ & $1.1\left(10^{-4}\right)$ & $3.0\left(10^{-5}\right)$ & $5.2\left(10^{-9}\right)$ \\
0.2 & $1.3\left(10^{-4}\right)$ & $5.5\left(10^{-5}\right)$ & $3.0\left(10^{-10}\right)$ & $1.3\left(10^{-4}\right)$ & $5.5\left(10^{-5}\right)$ & $2.6\left(10^{-9}\right)$ \\
0.3 & $8.1\left(10^{-5}\right)$ & $9.5\left(10^{-5}\right)$ & $9.5\left(10^{-11}\right)$ & $8.1\left(10^{-5}\right)$ & $9.6\left(10^{-5}\right)$ & $3.5\left(10^{-9}\right)$ \\
0.4 & $1.1\left(10^{-5}\right)$ & $1.2\left(10^{-4}\right)$ & $1.1\left(10^{-9}\right)$ & $1.1\left(10^{-5}\right)$ & $1.2\left(10^{-4}\right)$ & $1.2\left(10^{-9}\right)$ \\
0.5 & $4.3\left(10^{-5}\right)$ & $1.2\left(10^{-2}\right)$ & $1.3\left(10^{-9}\right)$ & $4.2\left(10^{-5}\right)$ & $1.2\left(10^{-4}\right)$ & $5.1\left(10^{-10}\right)$ \\
0.6 & $6.4\left(10^{-5}\right)$ & $9.3\left(10^{-5}\right)$ & $5.9\left(10^{-10}\right)$ & $6.4\left(10^{-5}\right)$ & $9.3\left(10^{-5}\right)$ & $9.9\left(10^{-10}\right)$ \\
0.7 & $5.2\left(10^{-5}\right)$ & $4.9\left(10^{-5}\right)$ & $1.1\left(10^{-9}\right)$ & $5.2\left(10^{-5}\right)$ & $4.8\left(10^{-5}\right)$ & $5.0\left(10^{-10}\right)$ \\
0.8 & $2.2\left(10^{-5}\right)$ & $2.3\left(10^{-5}\right)$ & $3.9\left(10^{-11}\right)$ & $2.2\left(10^{-5}\right)$ & $2.2\left(10^{-5}\right)$ & $5.9\left(10^{-11}\right)$ \\
0.9 & $1.6\left(10^{-5}\right)$ & $6.4\left(10^{-5}\right)$ & $1.0\left(10^{-9}\right)$ & $1.6\left(10^{-5}\right)$ & $6.4\left(10^{-5}\right)$ & $1.9\left(10^{-9}\right)$ \\
\hline
\end{tabular}

Note that $U_{P}^{e}, V_{P}^{e}$ represents absolute errors in $U(x, y), V(x, y)$, respectively, obtained using the proposed method.

\section{Conclusion and future work}

The method presented in this paper is a simple and highly accurate method for approximating a numerical estimate of coupled systems of fractional PDEs. We observe that the error of the approximation is much less than $10^{-5}$, which is an acceptable number. We believe that this paper will act as a basis for establishing other numerical schemes for much more complicated problems arising in the recent fields of biomedical and mechanical engineering and other branches of science.

Acknowledgement. We are grateful to the unknown reviewers for their useful comments and suggestions. Due to their helpful suggestions, the quality of the paper has been improved.

\section{References}

1. I. Aksikas, A. Fuxman, J. F. Forbes and J. Winkin, 'LQ control design of a class of hyperbolic PDE systems: application to fixed-bed reactor', Automatica 45 (2009) no. 6, 1542-1548.

2. A. Arikoglu and I. Ozkol, 'Solution of fractional differential equations by using differential transform method', Chaos Solitons Fractals 34 (2007) no. 5, 1473-1481.

3. F. AyAz, 'Solutions of the system of differential equations by differential transform method', Appl. Math. Comput. 147 (2004) 547-567.

4. C. Chen and C. HsiaO, 'Haar wavelet method for solving lumped and distributed parameter systems', IEE Press Contr. Theor. Appl. 144 (1997) 87-94.

5. M. Dehghan, J. Manafian and A. SaAdatmandi, 'Solving nonlinear fractional partial differential equations using the homotopy analysis method', Numer. Methods Partial Differential Equations 26 (2010) no. $2,448-479$.

6. M. Dehghan, J. Manafian and A. SaAdatmandi, 'The solution of the linear fractional partial differential equations using the homotopy analysis method', Z. Naturforsch. A 65a (2010) no. 11, 935-949.

7. M. Dehghan, M. Safarpoorand and M. AbBaszadeh, 'Two high-order numerical algorithms for solving the multi-term time fractional diffusion-wave equations', J. Comput. Appl. Math. 290 (2015) 174-195.

8. V. S. ERturk and S. Momani, 'Solving systems of fractional differential equations using differential transform method', J. Comput. Appl. Math. 215 (2008) 142-151.

9. M. R. Eslahchi and M. Dehghan, 'Application of Taylor series in obtaining the orthogonal operational matrix', Comput. Math. Appl. 61 (2011) no. 6, 2596-2604. 
10. K. Fackeldey and R. Krause, 'Multiscale coupling in function space weak coupling between molecular dynamics and continuum mechanics', Int. J. Numer. Methods Eng. 79 (2012) no. 12, 1517-1535.

11. G. B. Folland, Introduction to partial differential equations, 2nd edn (Princeton University Press, Princeton, NJ, 1995).

12. M. Gasea and T. Sauer, 'On the history of multivariate polynomial interpolation', J. Comput. Appl. Math. 122 (2000) 23-35.

13. R. Gorenflo, F. Mainardi, E. Scalas and M. Raberto, 'Fractional calculus and continuous time finance III', Math. Finance (2000) 171-180.

14. K. S. HEDRIH, 'Transversal creep vibrations of a beam with fractional derivative constitutive relation order. I-Partial fractional differential equation. II-Stochastic stability of the beam dynamic shape, under axial bounded noise excitation', Proceedings of Fourth International Conference on Nonlinear Mechanics (ICNM-IV), Shanghai, P.R. China (eds W. Z. Chien et al.; 2002) 584-595.

15. R. Hilfer, Applications of fractional calculus in physics (World Scientific Publishing Company, Singapore, 2000).

16. Y. Hu, Y. LuO and Z. Lu, 'Analytical solution of the linear fractional differential equation by Adomian decomposition method', J. Comput. Appl. Math. 215 (2008) 220-229.

17. R. W. Ibrahim, 'Solutions to systems of arbitrary-order differential equations in complex domains', Electron. J. Differential Equations 46 (2014) 1-13.

18. H. JAFARI and S. SEIFI, 'Solving a system of nonlinear fractional partial differential equations using homotopy analysis method', Commun. Nonlinear Sci. Numer. Simul. 14 (2009) 1962-1969.

19. R. KATICA and S. HEDRIh, 'Dynamics of multi-pendulum systems with fractional order creep elements', J. Theoret. Appl. Mech. 46 (2008) no. 3, 483-509.

20. R. Katica and S. Hedrin, 'Fractional order hybrid system dynamics', Proc. Appl. Math. Mech. 13 (2013) 25-26.

21. A. Kayedi-Bardeh, M. R. Eslahchi and M. Dehghan, 'A method for obtaining the operational matrix of the fractional Jacobi functions and applications', J. Vib. Control 20 (2014) no. 5, 736-748.

22. H. KhaliL and R. A. Khan, 'A new method based on Legendre polynomials for solutions of the fractional two-dimensional heat conduction equation', Comput. Math. Appl. 67 (2014) 1938-1953.

23. H. Khalil and R. A. Khan, 'A new method based on Legendre polynomials for solution of system of fractional order partial differential equations', Int. J. Comput. Math. 91 (2014) no. 12, 2554-2567.

24. H. Khalil and R. A. Khan, 'Extended spectral method for fractional order three-dimensional heat conduction problem', Prog. Fract. Differ. Appl. 1 (2015) no. 3, 165-185.

25. R. A. Khan and M. Rehman, 'Existence of multiple positive solutions for a general system of fractional differential equations', Commun. Appl. Nonlinear Anal. 18 (2011) 25-35.

26. A. A. Kilbas, H. M. Srivastava and J. Trujillo, Theory and applications of fractional differential equations (Elsevier Science, Amsterdam, 2006).

27. M. Lakestani, M. Dehghan and S. I. Pakchin, 'The construction of operational matrix of fractional derivatives using B-spline functions', Commun. Nonlinear Sci. Numer. Simul. 17 (2012) no. 3, 1149-1162.

28. Y. L. LI, 'Solving a nonlinear fractional differential equation using Chebyshev wavelets', Nonlinear Sci. Numer. Simul. 15 (2010) 2284-2292.

29. L.-L. Lin, Z.-Y. Li and B. Lin, 'Engineering waveguide-cavity resonant side coupling in a dynamically tunable ultracompact photonic crystal filter', Phys. Rev. B 72 (2005) 304-315.

30. S. Linge, J. Sundnes, M. Hanslien, G. T. Lines and A. Tveito, 'Numerical solution of the bidomain equations', Phil. Trans. Ser. A. Math. Phys. Eng. Sci. 367 (2009) 1931-1950.

31. F. Liu, V. ANH and I. Turner, 'Numerical solution of the space fractional Fokker-Planck equation', J. Comput. Appl. Math. 66 (2005) 209-219.

32. K. Maleknedjad, M. Shahrezaee and H. Khatami, 'Numerical solution of integral equation system of the second kind by block-pulse function', Appl. Math. Comput. 166 (2005) 15-24.

33. R. Metzler and J. Klafter, 'The random walk's guide to anomalous diffusion: a fractional dynamics approach', Phy. Rep. 339 (2000) no. 1, 1-77.

34. R. Metzler and J. Klafter, 'Boundary value problems for fractional diffusion equations', Phys. A: Stat. Mech. Appl. 278 (2005) 107-125.

35. A. A. Moghadam, I. Aksikas, S. Dubljevic and J. F. Forbes, 'LQ control of coupled hyperbolic PDEs and ODEs: application to a CSTR-PFR system', Proceedings of the 9th International Symposium on Dynamics and Control of Process Systems (DYCOPS 2010), Leuven, Belgium (eds M. Kothare et al.; 2010).

36. M. A. Mohamed and M. Sh. Torky, 'Solution of linear system of partial differential equations by Legendre multiwavelet and Chebyshev multiwavelet', Intl J. Sci. Innov. Math. Res. 2 (2014) no. 12, 966-976.

37. S. Nemati and Y. ORdokhani, 'Legendre expansion methods for the numerical solution of nonlinear $2 D$ Fredholm integral equations of the second kind', J. Appl. Math. Inform. 31 (2013) 609-621.

38. K. B. Oldham, 'Fractional differential equations in electrochemistry', Adv. Eng. Soft. 41 (2010) 9-12.

39. P. N Paraskevopolus, P. D. Saparis and S. G. Mouroutsos, 'The Fourier series operational matrix of integration', Int. J. Syst. Sci. 16 (1985) 171-176. 
40. V. PARThiban and K. Balachandran, 'Solutions of system of fractional partial differential equations', Appl. Appl. Math. 8 (2013) no. 1, 289-304.

41. I. Podlubny, Fractional differential equations (Academic Press, San Diego, CA, 1999).

42. M. RazzAGhi and S. Yousefi, 'The Legendre wavelets operational matrix of integration', Internat. J. Systems Sci. 32 (2001) 495-502.

43. M. RAZZAGHI and S. Yousefi, 'Sine-cosine wavelets operational matrix of integration and its application in the calculus of variation', Int. J. Syst. Sci. 33 (2002) 805-810.

44. M. Rehman and R. A. Khan, 'The Legendre wavelet method for solving fractional differential equation', Commun. Nonlinear Sci. Numer. Simul. 16 (2011) 4163-4173.

45. M. Rehman and R. A. Khan, 'A note on boundary value problems for a coupled system of fractional differential equations', Comput. Math. Appl. 61 (2011) 2630-2637.

46. G. Richard and P. R. R. SARMA, 'Reduced order model for induction motors with two rotor circuits', IEEE Trans. Energy Conv. 9 (1994) no. 4, 673-678.

47. Y. Rosikin and M. Shitikova, 'Application of fractional calculus for dynamic problems of solid mechanics', Amer. Soc. Mech. Eng. 63 (2010) 010801 1-52.

48. A. SaAdatmandi and M. Deghan, 'Numerical solution of a mathematical model for capillary formation in tumor angiogenesis via the tau method', Commun. Numer. Method. Eng. 24 (2008) 1467-1474.

49. A. SaAdatmandi and M. Deghan, 'A new operational matrix for solving fractional-order differential equation', Comput. Math. Appl. 59 (2010) 1326-1336.

50. K. Shah, H. Khalil and R. A. Khan, 'Investigation of positive solution to a coupled system of impulsive boundary value problems for nonlinear fractional order differential equations', Chaos Solitons Fractals 77 (2015) 240-246.

51. K. Shah, S. Zeb and R. A. Khan, 'Existence and uniqueness of solutions for fractional order $m$-point boundary value problems', Frac. Diff. Calc. 5 (2015) no. 2, 171-181.

52. K. Shah, A. Ali and R. A. Khan, 'Degree theory and existence of positive solutions to coupled systems of multi-point boundary value problems', Bound. Value Probl. 2016 (2016) no. 43, 1-12.

53. J. Sundnes, G. T. Lines, K. A. Mardal and A. Tveito, 'Multigrid block preconditioning for a coupled system of partial differential equations modeling the electrical activity in the heart', Comp. Method. Biomech. Biomed. Eng. 5 (2002) no. 6, 397-409.

54. J. Sundnes, G. T. Lines and A. Tveito, 'An operator splitting method for solving the bidomain equations coupled to a volume conductor model for the torso', Math. Biosci. 194 (2005) no. 2, 233-248.

55. P. J. Torvik and R. L. BAGLEY, 'On the appearance of fractional derivatives in the behaviour of real materials', J. Appl. Mech. 51 (1984) 294-298.

56. R. M. WALD, 'Construction of solutions of gravitational, electromagnetic or other perturbation equations from solutions of decoupled equations', Phy. Rev. Lett. 41 (1978) no. 4, 203-209.

57. Y. WANG and Q. FAN, 'The second kind Chebyshev wavelet method for solving fractional differential equation', Appl. Math. Comput. 218 (2012) 85-92.

58. M. WAZwAZ, 'The decomposition method applied to systems of partial differential equations and to the reaction diffusion Brusselator model', Appl. Math. Comput. 110 (2000) 251-264.

59. S. Wensheng, 'Computer simulation and modeling of physical and biological processes using partial differential equations', University of Kentucky Doctoral Dissertations, Lexington, KY, 2007.

Kamal Shah

Department of Mathematics

University of Malakand

Chakadara $\operatorname{Dir}(L)$

Khyber Pakhtunkhwa

Pakistan

kamalshah408@gmail.com

Rahmat Ali Khan

Department of Mathematics

University of Malakand

Chakadara $\operatorname{Dir}(L)$

Khyber Pakhtunkhwa

Pakistan

rahmat_alipk@yahoo.com

\author{
Hammad Khalil \\ Department of Mathematics \\ University of Education \\ (Attock Campus) \\ Punjab \\ Pakistan
}

hammad.khalil@ue.edu.pk 